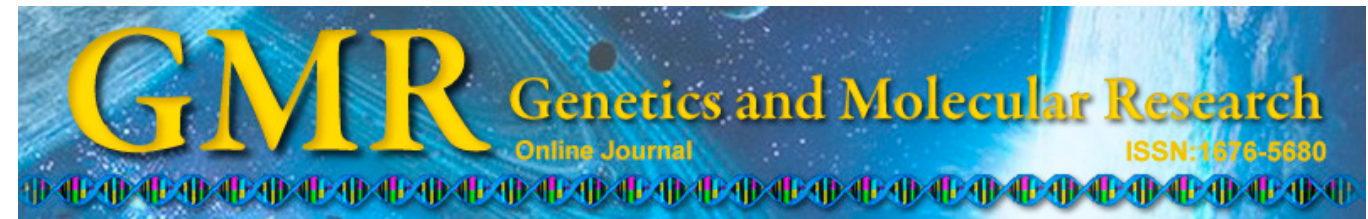

\title{
Genetic diversity and population structure of Eucommia ulmoides Oliver, an endangered medicinal plant in China
}

\author{
J. Yu ${ }^{1}$, Y. Wang ${ }^{2}$, L. Peng ${ }^{1}$, M. Ru ${ }^{2}$ and Z.S. Liang ${ }^{1,2}$ \\ ${ }^{1}$ College of Life Sciences, Northwest A\&F University, Yangling, China \\ ${ }^{2}$ The Center of Soil and Water Conservation and Ecoenvironmental Research, \\ Chinese Academy of Sciences, Yangling, China \\ Corresponding author: Z.S. Liang \\ E-mail: liangzs@ms.iswc.ac.cn
}

Genet. Mol. Res. 14 (1): 2471-2483 (2015)

Received November 3, 2014

Accepted March 23, 2015

Published March 30, 2015

DOI http://dx.doi.org/10.4238/2015.March.30.5

\begin{abstract}
Eucommia ulmoides Oliver, one of the tertiary relict species found only in China, is the only extant species of Eucommiaceae. Using inter-simple sequence repeat (ISSR) and sequence-related amplified polymorphism (SRAP) markers, we studied the genetic diversity and population genetic structure of 187 accessions from 17 E. ulmoides populations throughout its main distribution in China. A total of 65 bands were amplified using eight ISSR primers, of which 50 bands $(76.9 \%)$ were polymorphic. Meanwhile, another 244 bands were observed using eight SRAP primer combinations and 163 (66.8\%) of these were polymorphic. The analysis of molecular variation (AMOVA) indicated that 88.8 and $92.4 \%$ of the total variation resided within populations based on ISSR and SRAP analysis, respectively. Moreover, we found that the E. ulmoides populations were clustered into six distinct groups using ISSR and SRAP markers via the unweighted pair-group method (UPGMA). Furthermore, STRUCTURE analysis showed that these 17 populations could be classified into four groups using ISSR markers, but only two groups using SRAP markers. No
\end{abstract}


significant relevancy was observed between genetic and geographic distances among the sampled populations. The results of this study support the view that exchange of seeds among local farmers plays an important role in shaping the present genetic distribution pattern. "Core collection" is suggested for genetic diversity conservation of $E$. ulmoides in China.

Key words: Genetic diversity; Diversity conservation; ISSR; SRAP; Eucommia ulmoides Oliver

\section{INTRODUCTION}

Eucommia ulmoides Oliver, a typical elm-like deciduous woody plant, is a specific tertiary species that grows exclusively in China and is referred to as living fossil. More importantly, this plant is restricted to one species, one genus (Eucommia), and one family (Eucommiaceae) (Takeno et al., 2008). With the Chinese name Du-zhong, the bark of E. ulmoides has been applied as a traditional Chinese medicine (TCM) for more than 2000 years. The major effective constituents of $E$. ulmoides include types of lignans, iridoids, phenylpropanoids, flavonoids, polysaccharides, and terpenes (Dai et al., 2013). E. ulmoides possesses the potential for multiple pharmacological applications, including nourishment of the liver and kidney, strengthening of the muscles and bones, prevention of the high risk of abortion, hypertension, and diabetes (Kwan et al., 2003; National Pharmacopoeia Committee, 2010). Furthermore, Gutta-percha, an important industrial gum (Yan, 1989), can be also found in the leaves, bark, and seed shells of E. ulmoides (Tangpakdee et al., 1997).

As a second-category, state-protected endangered plant in China, E. ulmoides is situated in an endangered status in the wild. Considering both the biological conservation and the economic benefits, the Chinese government began to promote the cultivation of E. ulmoides in the early 1950s. Until now, a widespread artificial population has been mainly distributed in the Guizhou, Hubei, Henan, and Shaanxi provinces of China. E. ulmoides has rich genetic resources; however, previous studies have mainly focused on cultivation (Sun et al., 2013), Gutta-percha application (Suzuki et al., 2012), contents of active medicinal components (Dai et al., 2013), and pharmacological properties (Deyama et al., 2001). Thus, exploring the genetic diversity and population structure of E. ulmoides is very useful and important for its cultivation and production.

Using molecular markers to reveal polymorphism at the DNA level has been considered a powerful tool for evaluating the characteristics of plant genetic diversity. In previous studies, various molecular markers, such as inter-simple sequence repeats (ISSR) (Wu et al., 2011), random amplified polymorphic DNA (Wang et al., 2006), amplified fragment length polymorphism (Yao et al., 2012), and microsatellites (SSR) (Zhang et al., 2013) were used to detect the genetic diversity of E. ulmoides. Due to its superior reliability and reproducibility of bands, ISSR is regarded as a simple and efficient marker among these. Sequence-related amplified polymorphism (SRAP) markers, which specifically target open reading frames, have been widely used in population genetic studies of various plant species, including many medicinal plants (Song et al., 2010; Wang et al., 2012). However, to the best of our knowledge, SRAP markers have never been used in the genetic diversity analysis of E. ulmoides. 
The current prevailing hypothesis is that there are low levels of genetic differentiation among E. ulmoides populations, but each population may represent high genetic diversity. Yao et al. (2012) and Zhang et al. (2013) ascribed it to a high level of historical gene flow mediated by human activities. To test this hypothesis, more appropriate molecular makers are needed to investigate genetic variation of the more extant populations of E. ulmoides. Thus, we combined ISSR and SRAP markers to investigate the genetic composition of 187 accessions from 17 E. ulmoides populations across their main distribution. The aim of this study is to assess the genetic diversity and population structure of this endangered species, and to speculate a "core collection" that captures the most available genetic diversity of the species for conservation and breeding progress in the future.

\section{MATERIAL AND METHODS}

\section{Plant material}

We sampled 17 populations dispersed throughout the main distribution of E. ulmoides in China (Figure 1). The ages of individuals sampled ranged from 15 to 30 years. We collected over 1000 seeds from each of twenty trees selected in every population [twelve trees in the Ankang (AK) population]. The seeds of the same population were mixed together and seeded in the medicinal botanical garden at Northwest A\&F University, China. Fresh leaves of seedlings were collected for DNA extraction. Each population was positioned using a GPS and the details of the studied populations are described in Table 1.

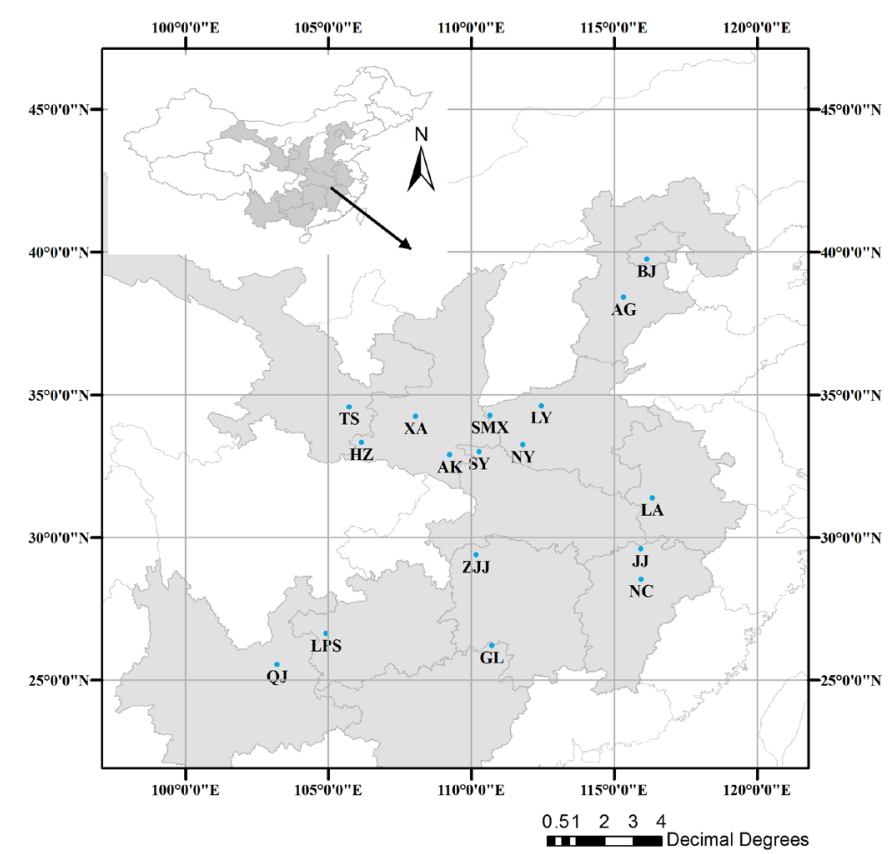

Figure 1. Geographical distribution of the 17 Eucommia ulmoides populations sampled in this study. Population abbreviations are provided in Table 1. 
Table 1. Details of the germplasm sources of Eucommia ulmoides.

\begin{tabular}{|c|c|c|c|c|c|c|}
\hline Population code & Location & Latitude $\left({ }^{\circ} \mathrm{N}\right)$ & Longitude $\left({ }^{\circ} \mathrm{E}\right)$ & Altitude (m) & Population size & Sample size \\
\hline$\overline{\mathrm{LY}}$ & Luoyang, Henan Province & $34^{\circ} 37^{\prime}$ & $112^{\circ} 27^{\prime}$ & 143 & 200 & 14 \\
\hline SMX & Sanmenxia, Henan Province & $34^{\circ} 17^{\prime}$ & $110^{\circ} 39^{\prime}$ & 926 & 2000 & 11 \\
\hline $\mathrm{HZ}$ & Hanzhong, Shaanxi Province & $33^{\circ} 20^{\prime}$ & $106^{\circ} 10^{\prime}$ & 687 & $>4000$ & 11 \\
\hline XA & Xi'an Xian, Shaanxi Province & $34^{\circ} 15^{\prime}$ & $108^{\circ} 03^{\prime}$ & 448 & 200 & 12 \\
\hline BJ & Beijing & $39^{\circ} 45^{\prime}$ & $116^{\circ} 08^{\prime}$ & 51 & 200 & 13 \\
\hline ZJJ & Zhangjiajie, Hunan Province & $29^{\circ} 24^{\prime}$ & $110^{\circ} 10^{\prime}$ & 389 & 900 & 9 \\
\hline GL & Guilin, Guangxi Province & $26^{\circ} 13^{\prime}$ & $110^{\circ} 43^{\prime}$ & 427 & 500 & 13 \\
\hline SY & Shiyan, Hubei Province & $33^{\circ} 01^{\prime}$ & $110^{\circ} 16^{\prime}$ & 698 & 1000 & 11 \\
\hline LA & Liu'an, Anhui Province & $31^{\circ} 24^{\prime}$ & $116^{\circ} 20^{\prime}$ & 124 & 900 & 13 \\
\hline $\mathrm{AG}$ & Anguo, Hebei Province & $38^{\circ} 25^{\prime}$ & $115^{\circ} 20^{\prime}$ & 32 & 1200 & 9 \\
\hline TS & Tianshui, Gansu Province & $34^{\circ} 35^{\prime}$ & $105^{\circ} 43^{\prime}$ & 1171 & 1500 & 9 \\
\hline LPS & Liupanshui, Guizhou Province & $26^{\circ} 39^{\prime}$ & $104^{\circ} 55^{\prime}$ & 1951 & 2000 & 14 \\
\hline $\mathrm{NC}$ & Nanchang, Jiangxi Province & $28^{\circ} 33^{\prime}$ & $115^{\circ} 57^{\prime}$ & 20 & 1000 & 8 \\
\hline QJ & Qujing, Yunnan Province & $25^{\circ} 33^{\prime}$ & $103^{\circ} 12^{\prime}$ & 2050 & 500 & 9 \\
\hline NY & Nanyang, Henan Province & $33^{\circ} 03^{\prime}$ & $111^{\circ} 15^{\prime}$ & 417 & 1200 & 9 \\
\hline JJ & Jiujiang, Jiangxi Province & $29^{\circ} 36^{\prime}$ & $115^{\circ} 56^{\prime}$ & 137 & 500 & 10 \\
\hline $\mathrm{AK}$ & Ankang, Shaanxi Province & $32^{\circ} 54^{\prime}$ & $109^{\circ} 14^{\prime}$ & 269 & 12 & 12 \\
\hline
\end{tabular}

\section{DNA extraction}

Total genomic DNA was extracted from approximately $100 \mathrm{mg}$ fresh leaves using the NuClean PlantGen DNA Kit (KangWei, China). The DNA was then suspended in TE buffer. DNA concentration and quality were evaluated by electrophoresis on a $1.0 \%$ agarose gel. DNA was stored at $-20^{\circ} \mathrm{C}$.

\section{PCR amplification}

A set of 100 ISSR primers (Isshiki et al., 2008) was synthesized according to the sequences obtained from the University of British Columbia, Canada. All 100 primers were screened for their amplification efficiency using six representative samples. According to the amplification efficiency and reproducibility, eight primer pairs (Table 2) were selected to test all populations. All samples were amplified at least three times if the initial amplification failed.

\begin{tabular}{|c|c|c|c|c|}
\hline ISSR primers sequences & Sequences $\left(5^{\prime} \rightarrow 3^{\prime}\right)$ & SRAP primer combinations & Forward primer sequences & Reverse primer sequences \\
\hline UBC825 & $(\mathrm{AC})_{8} \mathrm{~T}$ & Me2Em8 & TGAGTCCAAACCGGAGC & GACTGCGTACGAATTAGC \\
\hline UBC826 & $(\mathrm{AC})_{8}^{8} \mathrm{C}$ & Me5Em8 & TGAGTCCAAACCGGAAG & GACTGCGTACGAATTAGC \\
\hline UBC828 & $(\mathrm{TG})_{8}^{8} \mathrm{~A}$ & Me7Em4 & TGAGTCCAAACCGGTCC & GACTGCGTACGAATTTGA \\
\hline UBC848 & $(\mathrm{CA})_{8} \mathrm{RG}$ & Me8Em4 & TGAGTCCAAACCGGTGC & GACTGCGTACGAATTTGA \\
\hline UBC851 & $(\mathrm{GT})_{8}^{8} \mathrm{YG}$ & Me8Em9 & TGAGTCCAAACCGGTGC & GACTGCGTACGAATTACG \\
\hline UBC856 & $(\mathrm{AC})_{8} \mathrm{YT}$ & Me9Em5 & TGAGTCCAAACCGGACA & GACTGCGTACGAATTAAC \\
\hline UBC857 & $(\mathrm{AC})_{8}^{8} \mathrm{TA}$ & Me10Em5 & TGAGTCCAAACCGGAGA & GACTGCGTACGAATTAAC \\
\hline UBC881 & $\left(\mathrm{GGGTG}_{3}\right.$ & Me10Em9 & TGAGTCCAAACCGGAGA & GACTGCGTACGAATTACG \\
\hline
\end{tabular}

The PCR mixtures (20 $\mu \mathrm{L}$ total volume) contained $2.0 \mu \mathrm{L}$ 10X PCR buffer, $2.0 \mathrm{mM}$ $\mathrm{MgCl}_{2}, 200 \mathrm{mM}$ dNTPs, $1 \mathrm{mM}$ ISSR primers, $1 \mathrm{U}$ Taq DNA polymerase (KangWei), and approximately $10 \mathrm{ng}$ template DNA. PCR amplification was performed on an AB Applied Biosystem (Gene Company Limited, USA), using the following PCR program: 5 min of dena- 
turing at $94^{\circ} \mathrm{C}, 40$ cycles of three steps: $30 \mathrm{~s}$ of denaturing at $94^{\circ} \mathrm{C}, 30 \mathrm{~s}$ of annealing at $58^{\circ} \mathrm{C}$, and $1 \mathrm{~min}$ of elongation at $72^{\circ} \mathrm{C}$, with a final elongation step of $10 \mathrm{~min}$ at $72^{\circ} \mathrm{C}$. The PCR products were separated by electrophoresis on a 1.5\% agarose gel using $1 \mathrm{X}$ TBE buffer, $\mathrm{pH}$ 8.0, at room temperature, using a 5000-bp molecular size standard (KangWei). The gel was visualized using ethidium bromide staining.

SRAP analysis was carried out according to previously established protocols described by Li and Quiros (2001). SRAP primer combinations (100 pairs) were initially screened using six representative samples. Primer combinations were excluded if their banding patterns were difficult to score or if they failed to amplify consistently in all lines. Of these 100 SRAP primer pairs, eight primer combinations that consistently produced clear and diverse amplified bands were selected (Table 2). All samples were amplified at least three times if the initial amplification failed.

The PCR mixture ( $20 \mu \mathrm{L}$ total volume) contained $2.0 \mu \mathrm{L}$ 10X PCR buffer, $2.0 \mathrm{mM}$ $\mathrm{MgCl}_{2}, 200 \mathrm{mM}$ dNTPs, $0.5 \mathrm{mM}$ forward primer, $0.5 \mathrm{mM}$ reverse primer, $1 \mathrm{U}$ Taq DNA polymerase (KangWei), and approximately $20 \mathrm{ng}$ template DNA. PCR amplification was performed on an $\mathrm{AB}$ applied Biosystem (Gene Company Limited) using the following PCR program: 5 min of denaturing at $94^{\circ} \mathrm{C}, 5$ cycles of three steps: 1 min of denaturing at $94^{\circ} \mathrm{C}, 1$ min of annealing at $35^{\circ} \mathrm{C}$, and 2 min of elongation at $72^{\circ} \mathrm{C}$. In the following 35 cycles, the annealing temperature was increased to $50^{\circ} \mathrm{C}$, with a final elongation step of $8 \mathrm{~min}$ at $72^{\circ} \mathrm{C}$. The PCR products were separated on a $6 \%$ non-denaturing polyacrylamide gel and SRAP bands were stained using silver sequence DNA staining reagents, using a 5000-bp molecular size standard (KangWei).

\section{Data analysis}

Amplified bands were scored either as presence (1) or absence (0), and scored for a binary data matrix with polymorphic and reproducible bands. The matrix was then used for the following analysis: the percentage of polymorphic loci $(P P B)$, the effective number of alleles, observed number of alleles, gene flow $\left(N_{\mathrm{m}}\right)$, Shannon's information index $(I)$ (Lewontin, 1995), and Nei's gene diversity $(H)$ (Nei, 1973) were obtained by the software package POPGENE version 3.2 (Yeh et al., 1997). Analysis of molecular variance (AMOVA) was used to estimate the variance components and their significant levels of genetic variation within and among populations using GenAlEx version 6.5 (Peakall and Smouse, 2012) based on 999 permutations. A Mantel test (Nei, 1972) for geographic and genetic distances of population pairs was also employed using the same software to determine if a relationship existed between the two data matrices with 999 permutations.

To further examine the genetic relationships among accessions, cluster analysis based on the genetic similarity matrix was performed with the UPGMA (unweighted pair group method with arithmetic mean) method (Sneath and Sokal, 1973), using the SHAN function of NTSYS-pc version 2.1 (Rohlf, 2000). Population structure was determined using the modelbased program, STRUCTURE (Pritchard et al., 2000). To identify the number of populations $(K)$ capturing the major structure in the data, we used a burn-in period of 50,000 Markov Chain Monte Carlo iterations and 100,000 runs, with an admixture model following HardyWeinberg equilibrium, correlated allele frequencies, and independent loci for each run. Twenty independent runs were performed for each simulated value of $K$, ranging from 2 to 17 . The 
true $K$ value was determined using both an estimate of the posterior probability of the data for a given $K$ (Pritchard et al., 2000) and the Evanno $\Delta K$ (Evanno et al., 2005).

\section{RESULTS}

\section{Genetic diversity of $E$. ulmoides}

We surveyed 187 accessions from 17 E. ulmoides populations using eight ISSR primers (Table 3 ). A total of 65 bands were identified, of which 50 were polymorphic $(76.9 \%)$ with a minimum of 5 (UBC851) and a maximum of 12 (UBC848) bands per primer (Table 3 ). Representative banding patterns detected by the primer UBC-881 are shown in Figure 2. The size of amplified fragments ranged from 200 to $2000 \mathrm{bp}$ with an average of 8.1 fragments per primer. $P P B$ produced by each primer ranged from 40.0 (UBC-851) to $91.7 \%$ (UBC848). Genetic diversity (Table 4) was relatively low at the population level. PPB ranged from 24.6 to $60.0 \%$, with a mean of $42.6 \%$ (Table 4 ). The values of genetic diversity as estimated by $H$ varied from 0.085 to 0.245 and $I$ ranged from 0.130 to 0.356 . However, E. ulmoides populations had high levels of genetic diversity at the species level $(P P B=76.9 \% ; H=0.273$; $I=0.403)$.

Table 3. Polymorphism in Eucommia ulmoides revealed by inter-simple sequence repeat (ISSR) and sequencerelated amplified polymorphism (SRAP) markers.

\begin{tabular}{|c|c|c|c|c|c|c|c|}
\hline \multicolumn{4}{|c|}{ ISSR } & \multicolumn{4}{|c|}{ SRAP } \\
\hline Primer & Bands generated & $\mathrm{N}$ & $P P B(\%)$ & Primer combination & Bands generated & $\mathrm{N}$ & $P P B(\%)$ \\
\hline UBC825 & 9 & 8 & 88.9 & Me2Em8 & 15 & 7 & 46.7 \\
\hline UBC 826 & 8 & 4 & 50.0 & Me5Em8 & 46 & 31 & 67.4 \\
\hline UBC 828 & 7 & 6 & 85.7 & Me7Em4 & 23 & 14 & 60.9 \\
\hline UBC 848 & 12 & 11 & 91.7 & Me8Em4 & 27 & 14 & 51.9 \\
\hline UBC851 & 5 & 2 & 40.0 & Me8Em9 & 18 & 11 & 61.1 \\
\hline UBC 856 & 8 & 7 & 87.5 & Me9Em5 & 36 & 25 & 69.4 \\
\hline UBC857 & 9 & 7 & 77.8 & Me10Em5 & 35 & 27 & 77.1 \\
\hline UBC881 & 7 & 5 & 71.4 & Me10Em9 & 44 & 34 & 77.3 \\
\hline Total & 65 & 50 & 76.9 & Total & 244 & 163 & 66.8 \\
\hline Average & 8.1 & 6.3 & & Average & 30.5 & 20.4 & \\
\hline
\end{tabular}

$\mathrm{N}=$ number of polymorphic bands; $P P B=$ percentage of polymorphic bands.

Figure 2. Representative inter-simple sequence repeat banding patterns of primer UBC881 in Eucommia ulmoides. Population abbreviations are provided in Table 1.

The same accessions representing the 17 populations were then analyzed with 8 SRAP primer combinations (Table 3). These yielded a total of 244 fragments, of which 163 were polymorphic (66.8\%), with a minimum of 15 (Me2Em8) and a maximum of 46 (Me5Em8) bands per primer combination (Table 3). Representative banding patterns detected by the primer Me10Em5 are shown in Figure 3. The size of amplified fragments ranged from 100 to $5000 \mathrm{bp}$ with an average of 30.5 fragments per primer. $P P B$ produced by each primer pair ranged from 46.7 (Me2Em8) to $77.3 \%$ (Me10Em9). The inter-population genetic diversity (Table 4) was low. PPB ranged from 27.9 to $61.1 \%$, with a mean of $43.1 \%$ (Table 4). The 
values of genetic diversity as estimated by $H$ varied from 0.104 to 0.216 and $I$ ranged from 0.156 to 0.320 . In addition, E. ulmoides populations had high levels of genetic diversity at the species level $(P P B=66.8 \% ; H=0.228 ; I=0.345)$.

Table 4. Genetic diversity of Eucommia ulmoides populations reveled by combined inter-simple sequence repeat (ISSR) and sequence-related amplified polymorphism (SRAP) markers. Population abbreviations are provided in Table 1.

\begin{tabular}{|c|c|c|c|c|c|c|c|c|c|c|}
\hline \multirow[t]{2}{*}{ Population } & \multicolumn{5}{|c|}{ ISSR } & \multicolumn{5}{|c|}{ SRAP } \\
\hline & $N_{\mathrm{A}}$ & $N_{\mathrm{E}}$ & $H$ & $I$ & $P P B(\%)$ & $N_{\mathrm{A}}$ & $N_{\mathrm{E}}$ & $H$ & $I$ & $P P B(\%)$ \\
\hline $\mathrm{HZ}$ & 1.292 & 1.206 & 0.117 & 0.171 & 29.2 & 1.320 & 1.216 & 0.124 & 0.183 & 32.0 \\
\hline LY & 1.446 & 1.292 & 0.167 & 0.246 & 44.6 & 1.574 & 1.354 & 0.205 & 0.305 & 57.4 \\
\hline SMX & 1.600 & 1.440 & 0.245 & 0.356 & 60.0 & 1.586 & 1.328 & 0.197 & 0.298 & 58.6 \\
\hline XA & 1.338 & 1.234 & 0.133 & 0.196 & 33.8 & 1.352 & 1.248 & 0.140 & 0.206 & 35.2 \\
\hline BJ & 1.523 & 1.393 & 0.217 & 0.314 & 52.3 & 1.611 & 1.363 & 0.213 & 0.320 & 61.1 \\
\hline ZJJ & 1.477 & 1.325 & 0.186 & 0.274 & 47.7 & 1.279 & 1.177 & 0.104 & 0.156 & 27.9 \\
\hline GL & 1.415 & 1.307 & 0.171 & 0.248 & 41.5 & 1.312 & 1.196 & 0.116 & 0.173 & 31.2 \\
\hline SY & 1.554 & 1.395 & 0.222 & 0.322 & 55.4 & 1.574 & 1.373 & 0.216 & 0.319 & 57.4 \\
\hline LA & 1.523 & 1.371 & 0.209 & 0.305 & 52.3 & 1.430 & 1.237 & 0.143 & 0.217 & 43.0 \\
\hline $\mathrm{AG}$ & 1.477 & 1.326 & 0.187 & 0.274 & 47.7 & 1.549 & 1.366 & 0.208 & 0.307 & 54.9 \\
\hline TS & 1.415 & 1.295 & 0.166 & 0.242 & 41.0 & 1.451 & 1.306 & 0.175 & 0.258 & 45.1 \\
\hline LPS & 1.323 & 1.243 & 0.134 & 0.195 & 32.3 & 1.385 & 1.247 & 0.145 & 0.216 & 38.5 \\
\hline $\mathrm{NC}$ & 1.400 & 1.268 & 0.155 & 0.228 & 40.0 & 1.316 & 1.200 & 0.118 & 0.176 & 31.6 \\
\hline QJ & 1.323 & 1.219 & 0.126 & 0.186 & 32.3 & 1.307 & 1.205 & 0.118 & 0.175 & 30.7 \\
\hline NY & 1.508 & 1.367 & 0.207 & 0.301 & 50.8 & 1.508 & 1.334 & 0.192 & 0.285 & 50.8 \\
\hline JJ & 1.385 & 1.289 & 0.158 & 0.229 & 38.5 & 1.455 & 1.269 & 0.161 & 0.243 & 45.5 \\
\hline $\mathrm{AK}$ & 1.246 & 1.138 & 0.085 & 0.130 & 24.6 & 1.312 & 1.222 & 0.125 & 0.183 & 31.2 \\
\hline Mean & 1.426 & 1.300 & 0.170 & 0.248 & 42.6 & 1.431 & 1.273 & 0.159 & 0.236 & 43.1 \\
\hline At the species level & 1.723 & 1.476 & 0.273 & 0.403 & 76.9 & 1.693 & 1.380 & 0.228 & 0.345 & 66.8 \\
\hline
\end{tabular}

$N_{\mathrm{A}}=$ observed number of alleles; $N_{\mathrm{E}}=$ effective number of alleles; $H=$ Nei's gene diversity; $I=$ Shannon's Information index; $P P B=$ percentage of polymorphic loci.

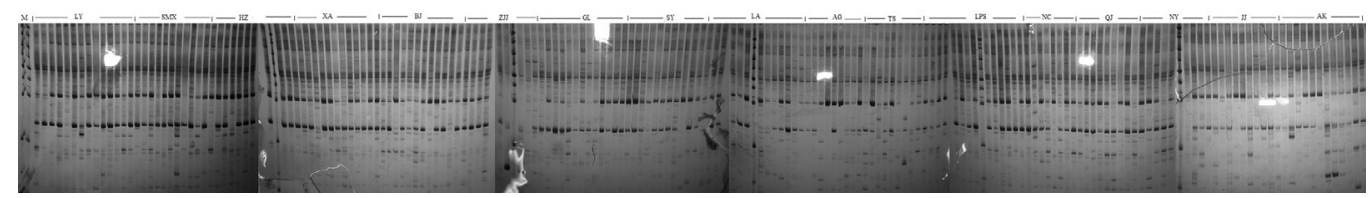

Figure 3. Representative sequence-related amplified polymorphism banding patterns of primer Me10Em5 in Eucommia ulmoides. Population abbreviations are provided in Table 1.

\section{Genetic differentiation and gene flow in $E$. ulmoides}

As revealed by the ISSR markers, the total gene diversity $(H T)$ and gene diversity within populations $(H S)$ were 0.271 and 0.170 , respectively. The coefficient of genetic differentiation $\left(G_{\mathrm{ST}}\right)$ was 0.374 . AMOVA analysis showed that $11.21 \%$ of the total gene diversity was found among the populations, whereas the remaining $88.79 \%$ of the total variation occurred within populations (Table 5), and $N_{\mathrm{m}}$ was 0.8357 . The SRAP analysis showed that $H T$ and $H S$ were 0.225 and 0.159 , respectively, and $G_{\mathrm{ST}}$ was 0.294 . The AMOVA analysis indicated that $7.62 \%$ of the total gene diversity was found among the populations, whereas the remaining $92.38 \%$ of the total variation occurred within the populations (Table 5), and $N_{\mathrm{m}}$ was 1.2007 . 
Table 5. Analysis of molecular variance (AMOVA) for Eucommia ulmoides populations revealed by combined inter-simple sequence repeat (ISSR) and sequence-related amplified polymorphism (SRAP) markers.

\begin{tabular}{llllrrrc}
\hline & SV & d.f. & SSD & MSD & VC & TVP & P \\
\hline ISSR & Among Pops & 16 & 196.66 & 12.291 & 0.983 & $11.21 \%$ & 0.001 \\
& Within Pops & 62 & 482.606 & 7.784 & 7.784 & $88.79 \%$ & \\
SRAP & Among Pops & 16 & 666.972 & 41.686 & 2.495 & $7.62 \%$ & 0.001 \\
& Within Pops & 62 & 1874.825 & 30.239 & 30.239 & $92.38 \%$ & \\
\hline
\end{tabular}

$\mathrm{SV}=$ source of variation; d.f. $=$ degrees of freedom; $\mathrm{SSD}=$ sum of squares; $\mathrm{MSD}=$ mean squares; VC $=$ variance component; TVP $=$ total variance percentage.

\section{Population structure and cluster analysis}

A dendrogram was plotted using UPGMA analysis derived from the ISSR profile data and is shown in Figure 4a. The genetic similarity (GS) coefficients among the 17 populations that were based on ISSR markers varied from 0.830 (between AK and the other populations) to 0.940 (between AK and the other populations). The 17 populations were placed into six clusters with a GS coefficient of 0.883 . The first major cluster (I) comprised ten populations. Clusters II and III included two populations each. The other 3 clusters (IV, V, and VI) had only one germplasm each, that is, the populations Qujing (QJ), Nanyang (NY), and AK, respectively. Two sub-clusters ( $\mathrm{Ia}, \mathrm{Ib})$ were identified within the first cluster at a GS coefficient of 0.889 . The first sub-cluster (Ia) comprised populations Hanzhong (HZ) and Xi'an Xian (XA), which were in the same province. The last eight populations were assigned to the second sub-cluster (Ib).

Based on SRAP analysis, the GS coefficients between all accessions ranged from 0.870 (between population AK and other populations) to 0.980 [between population Sanmenxia (SMX) and other populations]. The SRAP-based dendrogram also showed one major cluster (I) comprising eleven populations with a GS coefficient of 0.930 (Figure 4b). Cluster II consisted of populations Tianshui (TS) and NY. The populations Liupanshui (LPS), Jiujiang (JJ), XA, and AK appeared to be distinct from all other populations, each forming a single cluster (III, IV, $\mathrm{V}$, and VI), respectively. Cluster I was divided into three sub-clusters with a GS coefficient of 0.938 ; the first sub-cluster (Ia) comprised four populations, while six populations were assigned to the second sub-cluster (Ib). The population Zhangjiajie (ZJJ) alone formed sub-cluster Ic.
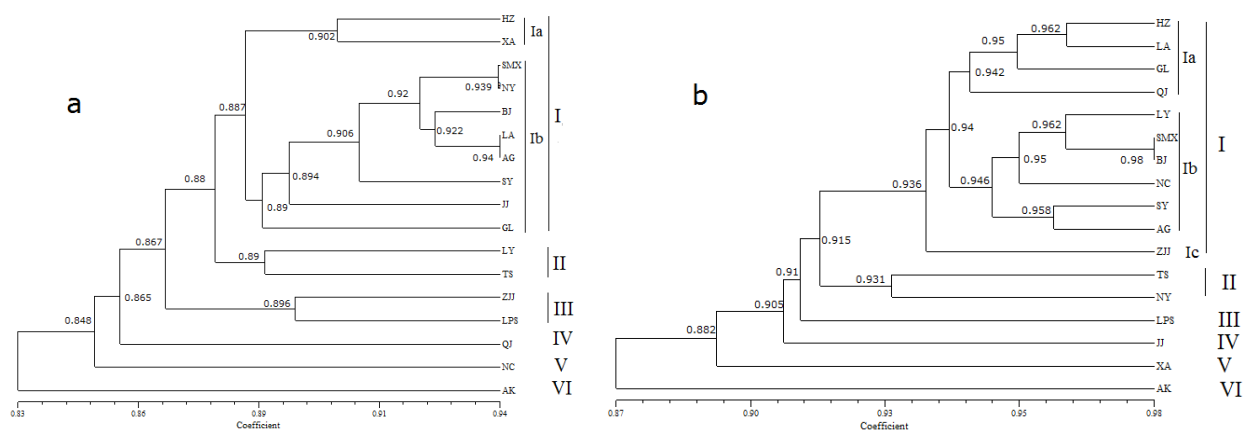

Figure 4. Dendrogram, constructed using the unweighted pair group method with arithmetic mean method, of the 17 Eucommia ulmoides populations sampled based on Nei's genetic distance, as revealed using inter-simple sequence repeat markers (a); sequence-related amplified polymorphism markers (b). The genetic similarity coefficients between populations obtained by cluster analyses are marked on branches and the x-axis. Population abbreviations are provided in Table 1. 
Following the method of Evanno (2005), the $\Delta K$ values were plotted against the $K$ numbers of the sub-groups. The maximum $\Delta K$ occurred at $K=4$ based on ISSR markers and $K=2$ based on SRAP markers (Figure 5). We divided the accessions into different sub-groups considering membership probabilities ( $\geq 0.25$ for ISSR, $\geq 0.50$ for SRAP). The STRUCTURE analysis (Figure 6a) indicated that the entire population could be divided into four groups based on the ISSR markers: group 1 comprised eight populations; group 2 consisted of the five populations SMX, Liu'an (LA), Guilin (GL), ZJJ, and QJ; populations Shiyan (SY) and Luoyang (LY) formed group 3, while populations HZ and NY comprised group 4. Based on the SRAP marker analysis, the 17 populations were divided into two groups (Figure 6b): group 1 included eleven populations, HZ, LY, Beijing (BJ), SY, Anguo (AG), TS, NY, LPS, JJ, XA, and AK; the other six populations [LA, GL, QJ, SMX, Nanchang (NC) and ZJJ] were clustered into group 2.
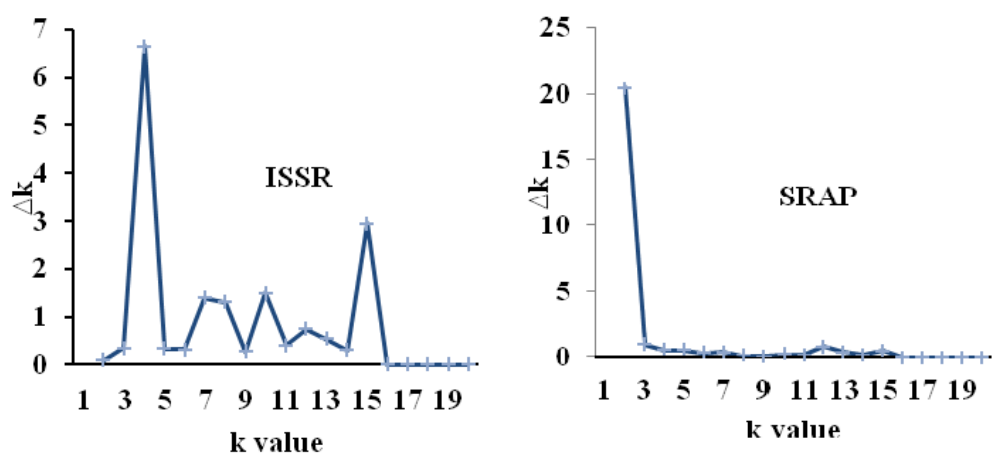

Figure 5. $\Delta K$ values for different numbers of Eucommia ulmoides populations assumed $(K)$ in the STRUCTURE analysis based on inter-simple sequence repeat markers (ISSR; left panel) and sequence-related amplified polymorphism markers (SRAP; right panel). Population abbreviations are provided in Table 1.
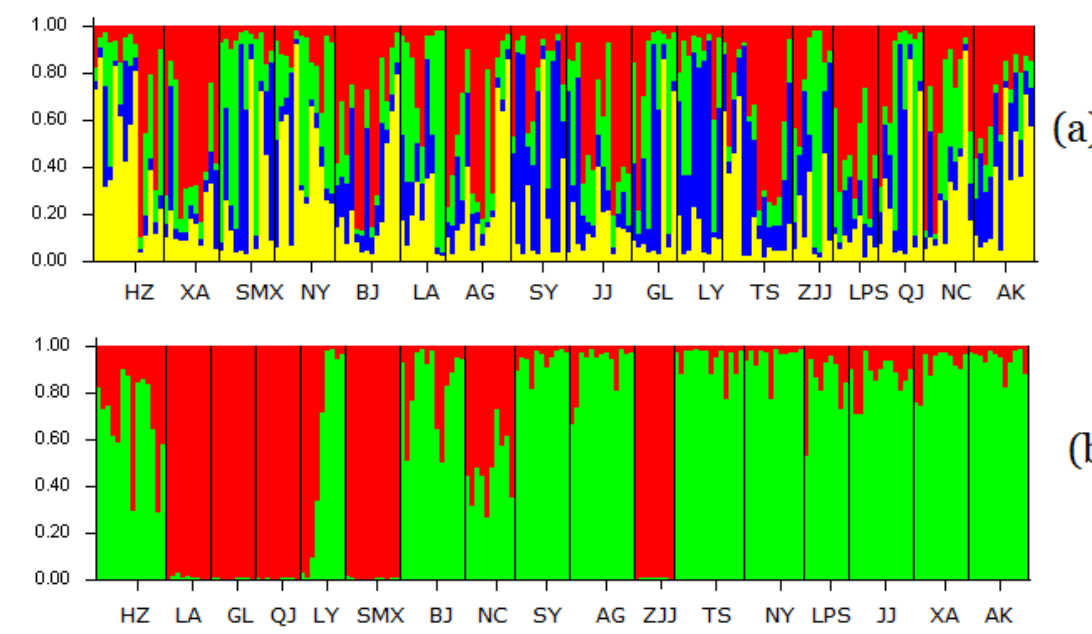

(b)

Figure 6. Population structure of Eucommia ulmoides prepared using the STRUCTURE program (Pritchard et al., 2000), as revealed using inter-simple sequence repeat markers (a); sequence-related amplified polymorphism markers (b). Population abbreviations are provided in Table 1. 


\section{DISCUSSION}

\section{Genetic diversity of $E$. ulmoides}

E. ulmoides, the only extant species of Eucommiaceae, is one of the oldest and most effective herbal medicines, and is widely used for treatment of hypertension (Dai et al., 2013). Hence, to better conserve and utilize this endangered species, it is necessary to gather as great a diversity of material as possible. Therefore, we collected samples from 17 populations, attempting to include the main distribution range of E. ulmoides in China. Eight ISSR primers and eight SRAP primer combinations were used to analyze the 187 E. ulmoides samples representing the 17 populations. ISSR analysis revealed high genetic diversity at the species level $(P P B=76.9 \% ; H=0.273 ; I=0.4034)$, but genetic diversity was relatively low at the population level $(P P B=42.63 \% ; H=0.1696 ; I=0.2482)$. The same result was observed based on the SRAP data, at the species level $P P B=66.8 \%, H=0.2276$, and $I=0.3447$, while at the population level $P P B=42.63 \%, H=0.1589$, and $I=0.2363$. Likewise, Wang et al. (2006), Wu et al. (2011), Yao et al. (2012), and Zhang et al. (2013) all revealed relatively high genetic diversity of E. ulmoides at the population level.

Both Yao et al. (2012) and Zhang et al. (2013) reported that the genetic diversity of the semi-wild population was greater than the cultivated population, which suggests that ongoing cultivation has reduced the genetic diversity of E. ulmoides. Our study showed that the SMX population had a relatively high genetic diversity $(P P B=60.0 \%$ for ISSR, $P P B=58.6 \%$ for SRAP), and this was attributed to hybridization, which may result in high levels of genetic variation in rare species (Leimu et al., 2006). Both ISSR and SRAP analysis indicated that the genetic diversity of the AK population was lower than the other populations $(P P B=24.6 \%$ and $31.2 \%$, respectively). However, only 12 individuals were found in the AK population, and the small population size may be the main factor for its low genetic diversity (Brzosko et al., 2011). Small populations are predicted to have reduced genetic diversity caused by genetic drift, founder effects, and accumulation of deleterious mutations (Young et al., 1996).

\section{Genetic differentiation of $E$. ulmoides populations}

Based on the values of $G_{\mathrm{ST}}(0.374$ for ISSR, 0.294 for SRAP), we observed relatively low genetic differentiation among the populations of E. ulmoides. The AMOVA analysis based on the ISSR and SRAP markers further indicated that 11.21 and $7.62 \%$ of the total gene diversity, respectively, was found among populations (Table 2). Yao et al. (2012) indicated that low to moderate levels of genetic differentiation among E. ulmoides populations may result from the relatively high gene flow among the studied populations.

In the present study, $N_{\mathrm{m}}$ among the populations determined by ISSR and SRAP analysis was 0.836 and 1.201 , respectively. The value of $N_{\mathrm{m}}>1$ indicates that no significant genetic differentiation existed among populations, and this level of migration would not restrain the continued divergence among the populations (Slatkin, 1987). Thus, the results of the SRAP analysis indicate that no significant genetic differentiation exists among populations. In addition, according to the Mantel test, no significant correlation between genetic distance and geographical distance was detected in this study. Similar results were also found in some other studies of E. ulmoides (Wang et al., 2006; Yao et al., 2012; Zhang et al., 2013). However, the 
results of the ISSR analysis indicate just the opposite. Wu et al. (2011) also suggested that gene flow in E. ulmoides was low, which was based on an $N_{\mathrm{m}}$ value of 0.412 using ISSR markers.

Lower gene flow is commonly found in rare or endangered species because the distribution and population size of these species are often dramatically reduced. Nevertheless, our SRAP analysis in E. ulmoides indicated that this endemic plant has a high level of gene flow. The mode of pollen and seed dispersal, which determine gene flow among populations, may partly account for the status of gene flow. E. ulmoides is a wind pollinated and outcrossing species and the distances among populations are too large for pollen dispersal. Thus, seeds appear to be the main genetic material exchanged from one geographic region to another. Local farmers collect seeds of $E$. ulmoides randomly and mix them together before planting. Occasionally, the germplasm may be dispersed to other places by farmers. This frequent exchange of seeds further improves the maintenance of genetic variation (Guo et al., 2007).

\section{Cluster analysis}

Similar results were obtained from the STRUCTURE analysis using ISSR and SRAP markers. According to the SRAP analysis, the populations were clustered into two main groups, but the HZ, LY, and NC populations displayed some degree of mixed ancestry. The NC population was especially admixed and exhibited inheritance from two different ancestors; accessions in the NC population were similar to both main groups. This result was further confirmed by ISSR analysis, with the LY and NC populations clustered into group 3 and group 4 , respectively. There was no significant correlation between the ISSR and SRAP dendrograms, except for the AK population, which formed a genetically distinct group. A Mantel test showed no significant relationship between genetic and geographic distances among the sampled populations ( $r=0.022$ for ISSR, $r=-0.046$ for SRAP). This corroborates previous reports showing there was no statistically significant correlation between the pairwise genetic distance and the corresponding geographic distance among the populations (Wu et al., 2011; Yao et al., 2012).

\section{Implications for conservation}

A long cultivation history of E. ulmoides has played an important role in shaping the present genetic distribution pattern. In traditional forestry breeding, conventional breeding of E. ulmoides has mainly focused on the selection of promising plants from existing natural populations. These selected plants were propagated vegetatively and released as clones ( $\mathrm{Li}$ et al., 2014). However, clonal reproduction for desirable characteristics narrows genetic variation and is not appropriate for conservation because this material includes only a portion of the gene pool. To protect the TCM germplasm resource, the "medicinal plant core collection" conception was proposed recently (Liu et al., 2012). Seed collection may be a better consideration at present and in the future for the germplasm conservation of E. ulmoides.

The main objective of genetic resource conservation is to maintain high levels of genetic variability. According to our present study, the SMX, BJ, LY, SY, LA, AG, and NY populations possess higher genetic variation than the mean, suggesting that these seven populations should be given high priority in conservation. These seven populations were closely clustered together in the ISSR and SRAP dendrograms, while the dendrograms all showed that the AK population formed a genetically distinct group. Thus, the AK population should be given pri- 
ority in germplasm collection, and a proper conservation measure should be instituted for its low genetic diversity at the inter-population level, which is mainly due to its small population size. A decreased population size and increased isolation strengthen the effects of genetic drift, which is capable of reducing the variation within populations and increasing the differentiation among populations ( $\mathrm{Li}$ et al., 2005). Based on the STRUCTURE analysis, the HZ, LY, and $\mathrm{NC}$ populations are also important for conservation programs. Furthermore, most work by conservationists on medicinal plants could be achieved by cooperating with the people who own, manage, or make use of these species (Hamilton, 2004). Most E. ulmoides populations are cultivated, and the cultivators should be encouraged to protect this highly rare tree species, not only for economic benefits but also for the responsibility of protecting endangered plants.

In conclusion, our results based on ISSR and SRAP analysis indicate that these 17 cultivated populations of $E$. ulmoides exhibit high genetic diversity at the species level, with a relatively lower genetic diversity at the population level. Additionally, a low level of interpopulation genetic differentiation and a high level of intra-population genetic differentiation were found in our present study, which is supported by a moderate level of gene flow. Exchange of seeds among local farmers has played an important role in shaping the present genetic distribution pattern. Based on our research, several suggestions were proposed for genetic diversity conservation of E. ulmoides in China.

\section{Conflicts of interest}

The authors declare no conflict of interest.

\section{ACKNOWLEDGMENTS}

Research supported by the Seeds and Seedlings Quality Standard of Du-zhong (\#2012ZX09304006), which is the sub-task of major Chinese National Science and Technology Special Funds "Quality Standard Platform of Planting (Breeding) Seeds and Seedlings of Traditional Chinese Medicine".

\section{REFERENCES}

Brzosko E, Wróblewska A, Tałałaj I and Wasilewska E (2011). Genetic diversity of Cypripedium calceolus in Poland. Plant Syst. Evol. 295: 83-96.

Dai X, Huang Q, Zhou B, Gong Z, et al. (2013). Preparative isolation and purification of seven main antioxidants from Eucommia ulmoides Oliv. (Du-zhong) leaves using HSCCC guided by DPPH-HPLC experiment. Food Chem. 139: 563-570.

Deyama T, Nishibe S and Nakazawa Y (2001). Constituents and pharmacological effects of Eucommia and Siberian ginseng. Acta Pharmacol. Sin. 22: 1057-1070.

Evanno G, Regnaut S and Goudet J (2005). Detecting the number of clusters of individuals using the software STRUCTURE: a simulation study. Mol. Ecol. 14: 2611-2620.

Guo HB, Lu BR, Wu QH, Chen JK, et al. (2007). Abundant genetic diversity in cultivated Codonopsis pilosula populations revealed by RAPD polymorphisms. Genet. Resour. Crop Evol. 54: 917-924.

Hamilton AC (2004). Medicinal plants, conservation and livelihoods. Biodivers. Conserv. 13: 1477-1517.

Isshiki S, Iwata N and Khan MMR (2008). ISSR variations in eggplant (Solanum melongena L.) and related Solanum species. Sci. Hortic. 117: 186-190.

Kwan CY, Chen CX, Deyama T and Nishibe S (2003). Endothelium-dependent vasorelaxant effects of the aqueous extracts of the Eucommia ulmoides Oliv. leaf and bark: implications on their antihypertensive action. Vasc. Pharmacol. 40: 
229-235.

Leimu R, Mutikainen P, Koricheva J and Fischer M (2006). How general are positive relationships between plant population size, fitness and genetic variation? J. Ecol. 94: 942-952.

Lewontin RC (1995). The apportionment of human diversity. In: Evolutionary biology: volume 6 (Dobzhansky T, Hecht MK and Steere WC, eds.). Springer, USA, 381-398.

Li G and Quiros CF (2001). Sequence-related amplified polymorphism (SRAP), a new marker system based on a simple PCR reaction: its application to mapping and gene tagging in Brassica. Theor. Appl. Genet. 103: 455-461.

Li Y, Wang D, Li Z, Wei J, et al. (2014). A molecular genetic linkage map of Eucommia ulmoides and quantitative trait loci (QTL) analysis for growth traits. Int. J. Mol. Sci. 15: 2053-2074.

Li YY, Chen XY, Zhang X, Wu TY, et al. (2005). Genetic differences between wild and artificial populations of Metasequoia glyptostroboides: implications for species recovery. Conserv. Biol. 19: 224-231.

Liu XG, Huang LQ, Yuan QJ, Zhang YQ, et al. (2012). Construction of medicinal plant core collection based on molecular phylogeography. Zhongguo Zhong Yao Za Zhi 37: 692-698.

National Pharmacopoeia Committee (2010). Chinese Pharmacopoeia. China Medical Science Press, Beijing, 161-162.

Nei M (1972). Genetic distance between populations. Am. Nat. 3: 283-292.

Nei M (1973). Analysis of gene diversity in subdivided populations. Proc. Natl. Acad. Sci. U. S. A. 70: 3321-3323.

Peakall R and Smouse PE (2012). GenAlEx 6.5: genetic analysis in Excel. Population genetic software for teaching and research - an update. Bioinformatics 28: 2537-2539.

Pritchard JK, Stephens M and Donnelly P (2000). Inference of population structure using multilocus genotype data. Genetics 155: 945-959.

Rohlf JF (2000). NTSYS-pc, Numerical taxonomy multivariate analysis system. Version 2.1. Applied Biostatistics Inc., New York

Slatkin M (1987). Gene flow and the geographic structure of natural populations. Science 236: 787-792.

Sneath PHA and Sokal RR (1973). Numerical taxonomy: the principles and practice of numerical classification. WF Freeman and Co., San Francisco.

Song Z, Li X, Wang H and Wang J (2010). Genetic diversity and population structure of Salvia miltiorrhiza Bge in China revealed by ISSR and SRAP. Genetica 138: 241-249.

Sun Z, Li F, Du H, Zhu J, et al. (2013). A novel silvicultural model for increasing biopolymer production from Eucommia ulmoides Oliver trees. Ind. Crop Prod. 42: 216-222.

Suzuki N, Uefuji H, Nishikawa T, Mukai Y, et al. (2012). Construction and analysis of EST libraries of the transpolyisoprene producing plant, Eucommia ulmoides Oliver. Planta 236: 1405-1417.

Takeno S, Bamba T, Nakazawa Y, Fukusaki E, et al. (2008). Quantification of trans-1,4-polyisoprene in Eucommia ulmoides by fourier transform infrared spectroscopy and pyrolysis-gas chromatography/mass spectrometry. J. Biosci. Bioeng. 105: 355-359.

Tangpakdee J, Tanaka Y, Shiba KI, Kawahara S, et al. (1997). Structure and biosynthesis of trans-polyisoprene from Eucommia ulmoides. Phytochemistry 45: 75-80.

Wang AQ, Huang LQ, Shao AJ, Cui GH, et al. (2006). Genetic diversity of Eucommia ulmoides by RAPD analysis. Zhongguo Zhong Yao Za Zhi 31: 1583-1586.

Wang XM, Hou XQ, Zhang YQ, Yang R, et al. (2012). Genetic diversity of the endemic and medicinally important plant Rheum officinale as revealed by inter-simple sequence repeat (ISSR) markers. Int. J. Mol. Sci. 13: 3900-3915.

Wu MQ, Chen SL, Wang MX and Yan SZ (2011). An analysis of the genetic diversity and genetic structure of Eucommia ulmoides using inter-simple sequence repeat (ISSR) markers. Afr. J. Biotechnol. 10: 19505-19513.

Yan R (1989). An age-old and young natural polymer - gutta percha. Chin. Polymer Bull. 2: 39-43.

Yao X, Deng J and Huang H (2012). Genetic diversity in Eucommia ulmoides (Eucommiaceae), an endangered traditional Chinese medicinal plant. Conserv. Genet. 13: 1499-1507.

Yeh FC, Yang R-C, Boyle TBJ, Ye ZH et al. (1997). POPGENE, the user-friendly shareware for population genetic analysis. Molecular Biology and Biotechnology Centre, University of Alberta, Canada.

Young A, Boyle T and Brown T (1996). The population genetic consequences of habitat fragmentation for plants. Trends. Ecol. Evol. 11: 413-418.

Zhang J, Xing C, Tian H and Yao X (2013). Microsatellite genetic variation in the Chinese endemic Eucommia ulmoides (Eucommiaceae): implications for conservation. Bot. J. Linnean Soc. 173: 775-785. 